\title{
El modelo organizativo de la Economía del Bien Común y su comparación con otros enfoques de la sostenibilidad
}

\section{Joan Ramon Sanchis Palacio, Vanessa Campos Climent, Ana Ejarque Catalá}

RESUMEN: Son cada vez más las empresas que están adoptando la sostenibilidad como un enfoque estratégico que garantiza la supervivencia a largo plazo. Más allá de la Responsabilidad Social (RS), se impone un modelo empresarial basado en la triple dimensión económica, social y ambiental, que de acuerdo con el enfoque de la creación de valor compartido de Porter y Kramer, incluya la RS dentro del corazón de la estrategia de la empresa. El modelo de la Economía del Bien Común (EBC), que nace en el centro de Europa en el año 2010 de la mano del profesor de la Universidad de Viena Christian Felber, propone una herramienta, el Balance del Bien Común (BBC), que puede facilitar la implantación de la Sostenibilidad Corporativa en cualquier tipo de organización (pública o privada). El BBC permite medir el impacto social y ambiental de las empresas a través de una serie de variables e indicadores, relacionando cinco valores clave (dignidad humana, solidaridad y justicia social, sostenibilidad ecológica y transparencia y co-participación) con los diferentes stakeholders o grupos de interés. El objetivo de este trabajo consiste en comparar el modelo de la EBC con el enfoque de la Creación de Valor Compartido (CVC) de Porter y Kramer y con la Responsabilidad Social Corporativa, con el objeto de destacar sus principales aportaciones y particularidades.

PALABRAS CLAVE: Economía del Bien Común, Sostenibilidad, Valor Compartido, Balance del Bien Común.

CLAVES ECONLIT: A13, B55, M10, M14.

Cómo citar este artículo/How to cite this article: SANCHIS, J.R., CAMPOS, V., EJARQUE, A. (2021): "El modelo organizativo de la Economía del Bien Común y su comparación con otros enfoques de la sostenibilidad”, CIRIEC-España, Revista de Economía Pública, Social y Cooperativa, 101, 143-163. DOI: 10.7203/CIRIEC-E.101.16399.

Correspondencia: Joan Ramon Sanchis Palacio, Universitat de València, joan.r.sanchis@ uv.es, ORCID: 0000-0002-2475-3299; Vanessa Campos Climent, Universitat de València, vanessa. campos@uv.es, ORCID: 0000-0002-9561-7761; Ana Ejarque Catalá, Universitat de València, ana.t.ejarque@uv.es, 0RCID: 0000-0002-7346-3888. 


\section{Expanded abstract}

\section{The organizational model of the Economy for the Common Good and its comparison with other approaches to Sustainability}

\section{Objective}

This paper aims to analyze the Economy of the Common Good (ECG) model as an appropriate model for those companies that seek sustainability and base their behavior on ethics and the human dimension simultaneously. To do so, the authors compare the ECG model with the Shared Value Creation (CVC) approach and with Corporate Social Responsibility (RSC), in order to highlight its main contributions and particularities.

\section{Approach/methodology}

Christian Felber (Austrian sociologist and political activist) presented in 2008, a document entitled "New values for the Economy". In this document, he raises the bases for an alternative economic system to capitalism and communism. To do so, he had the support of a group of Austrian entrepreneurs. Thus, giving birth to the new economic and social model known as the Economy for the Common Good. Moreover, in 2010 he published the book entitled "Economy for the Common Good".

It is important to realize that the ECG model is being applied in close to 2,000 firms located in 30 countries worldwide, including Europe, North America, and Latin America. In fact, on December 31, 2017, there were already 657 companies throughout Europe (in 12 different countries) that were implementing the EBC organizational model. Another key point is the Opinion of the Economic and Social Committee on the Economy for the Common Good, which recognizes the ECG model as a sustainable economic model geared towards social cohesion.

In order to achieve the research objectives, the authors analyze the theoretical foundations of the CVC and its relationship with Corporate Sustainability (SC), and secondly, we present the ECG's bases.

Taking into consideration the definition of CVC, firms can create economic value and, simultaneously, create value for society by trying to solve its needs and challenges. Therefore, companies need to go beyond CSR, since these social responsibility policies place CVC as something peripheral to the company's strategy. This means these policies are used by companies as a way to seek social legitimacy and increase the short-term income statement.

Under those circumstances, SC arises from the relationship RSC and Sustainable Development as an inclusive business approach in which the different firms' stakeholders are integrat- 
ed into its development with the purpose of creating long-term value. This is to create value from an economic, social and environmental point of view. Notwithstanding, both models do not take into consideration the ethical behavior of companies.

By its part, the ECG model bases its principles and values not only on the economic and financial benefits, but on the need to measure social and environmental impacts. These values are 1) human dignity, 2) solidarity and social justice, 3) environmental sustainability, and 4) transparency and co-determination. Thus, these values are related to ethics and humanism, and also to the Sustainable Development Goals. In the light of this, firms working under the ECG framework prioritize social goals over financial and economic goals.

With this in mind, the ECG employs the Common Good Balance Sheet (BBC) as the tool to guide and measure the contribution of the business to the common good in terms of economic, social, and environmental impacts. Thus, the BBC takes a set of indicators as a starting point and works as an integrated report, is that is works as a source of information related to sustainability concerns for both internal and external stakeholders. Moreover, the BBC is addressed to any type of organization such as public administrations, firms, non-profit organizations, and even communities, including MSMEs, as the model counts with a simplified version designed for MSMEs.

Additionally, the $\mathrm{BBC}$ employs the Common Good Matrix (MBC) as a strategic matrix by connecting the firm's behavior regarding the general principles and values of human to the stakeholders grouped into five groups: 1)suppliers, 2)owners, equity, and financial services providers, 3) employees, 4) customers and business partners, and 5)social environment, thus resulting in 20 items. Then, each item is assigned with a maximum score of 50 points. Therefore, the maximum score for a company applying the MBC is 1,000 points.

Seeing that, one could assume that both the EBC model and the CVC approach highlight that sustainable companies are those capable of creating economic, social and environmental value. Hence, contrary to works that indicate companies' economic cost when applying CSR, both, CVC and the EBC model, point out that creating social and environmental value can also be beneficial for companies. In this sense, social responsibility actions must be understood, not as a cost, but as a real investment. This is, social and environmental value can lever a positive impact on the financial results of the company in the long term.

Although this may be true, different from the ECG model, the CVC approach does not propose to replace competition with cooperation. To put it differently, the ECG model considers cooperation as a fundamental factor when implementing the strategy while from the CVC point of view, competition remains the key to business success.

By all before pointed, the authors identify the ECG model as the closest to SC. Indeed, SC highlights the importance of ensuring the survival of future generations by means of environment conservation and social and human values' recovery. In this sense, the ECG model is a holistic and integrated model that ensures the contribution to SC. 


\section{Results/implications}

The first thing to remember is that enterprises are one of the key agents in the operation of the economy. For this reason, they must focus their efforts to social development through social and environmental creation, in addition to the creation of financial and economic value. With this intention, the ECG model, when applied to the business sphere, clearly contributes to the implementation of business models that drive to SC. In like manner, the ECG model can also become a marketing tool. In this sense, it is relevant to analyze the motivating factors of ECG firms when implementing the ECG model.

In order to get a better understanding, the authors propose the $\mathrm{MBC}$ and the $\mathrm{BBC}$ as tools that facilitate the management and monitoring of firms' behavior in terms of social and environmental concerns. Besides, the ECG model allows its implementation by any type of organization. As a matter of fact, at the end of 2017, close to 650 European enterprises were working under the ECG framework, even though its novel implementation.

\section{Practical conclusions and original value}

The ECG model not only provides a tool to measure social and environmental value for firms, but it is also a method to improve the management process, thus driving enterprises towards SC. Certainly, the BBC allows quantifying social and environmental variables, hence including within its operation such diverse approaches as feminist economics, circular economy, sustainable economy, or democratic economy, among others.

Given these points, the ECG model is a holistic business model focused on sustainability. As a result, it is suitable to achieve SC due to 1) the ECG model allows its implementation by any type of organization, 2) it allows quantifying social and environmental variables. In other words, the ECG model is a reliable and valid measurable model. 3) It enables the variables and indicators' adaptation to each organization, thus providing flexibility, and 4) it is a dynamic model since allows continuous improvement measures from a strategic perspective. Henceforth, future research should analyze the evolution and implementation degree achieved by the ECG model within a short time.

Keywords: Economy for the Common Good, Sustainability, Shared Value, Sheet for the Common Good. 


\section{Introducción}

Los excesos del sistema capitalista y los fallos del mercado son factores que han provocado el surgimiento de diferentes enfoques o teorías que intentan ofrecer alternativas al sistema desde una visión crítica de la economía ortodoxa y bajo una perspectiva más humana y social (Chaves Ávila y Monzón Campos, 2018). Aunque el cuestionamiento del sistema capitalista ha sido algo habitual desde sus inicios (Socialismo utópico, Marxismo), la mayoría de estos enfoques surgen sobre todo a partir de los inicios del Siglo XX (Sen, 1999; Chomsky, 2002; Zamagni, 2007) y se extienden con fuerza desde el estallido de la crisis financiera en 2008 (Rodrik, 2011; Jackson, 2011; Krugman, 2012).

Algunos de estos enfoques intentan suavizar o paliar parte de las externalidades provocadas por el capitalismo, como es el caso de la Economía Social, el Tercer Sector, la Economía Solidaria, la Economía Sostenible o la Responsabilidad Social Empresarial, entre otros (Chaves Ávila y Monzón Campos, 2018; Sanchis Palacio y Campos Climent, 2019; Campos Climent et al., 2020). Sin embargo, los resultados de todos estos modelos han sido parciales y locales, de manera que no han resuelto las graves contradicciones que genera el sistema capitalista y las desigualdades económicas y sociales han seguido aumentando de manera constante (Entrena Jiménez y Martínez Rodríguez, 2013).

Ante el panorama descrito, se hace necesario encontrar un nuevo modelo económico y social, total o parcial, de cariz más humano y social, que a la vez sea capaz de garantizar la democracia y la libertad. La crisis del 2008 ha tenido de positivo que ha abierto nuevas posibilidades a la economía crítica y alternativa, rompiendo así el pensamiento único del modelo neoliberal. Los movimientos de protesta como el 15M han favorecido cambios sociales y económicos, de manera que la sociedad civil está ofreciendo alternativas viables a los problemas del capitalismo. El uso de las redes sociales y de las tecnologías de la información y la comunicación ha ayudado a implementar nuevas formas económicas más justas, equitativas y solidarias como la economía colaborativa, la economía circular y la banca ética y social, entre otras (Entrena Jiménez y Martínez Rodríguez, 2013; Chaves Ávila y Monzón Campos, 2018).

En este contexto, se hace necesario consolidar un modelo económico y social alternativo sólido y aglutinador de todas estas tendencias (Sanchis Palacio y Campos Climent, 2019). El sociólogo y activista político austríaco Christian Felber presenta en el 2008 el documento Nuevos valores para la economía (Felber, 2008), en el que plantea las bases para un sistema alternativo al capitalismo y al comunismo; y con el apoyo de un grupo de empresarios/as austríacos/as es capaz de describir un nuevo modelo económico y social denominado Economía del Bien Común (EBC), que se recoge en su libro más conocido publicado con el mismo nombre en el $2010^{1}$. Así, el modelo de la EBC ha conseguido ser reconocido como un modelo alternativo por una parte importante de la sociedad, en relativamente poco espacio de tiempo, de manera que a 31 de diciembre de 2017 eran ya 657 las empresas en toda Europa (en 12 países diferentes)

1. La edición original en alemán data del año 2010, aunque su traducción a otras lenguas, entre ellas el castellano, es publicada en 2012 (Felber, 2012). Existe una versión actualizada de su libro de 2015. 
que estaban implementando el modelo organizativo de la EBC (Felber et al., 2019; Campos Climent et al., 2019). También la Unión Europea reconoce el modelo a partir del Dictámen del Consejo Económico y Social Europeo publicado en 2016 sobre "La Economía del Bien Común: un modelo económico sostenible orientado a la cohesión social". El modelo, sin llegar a rechazar el mercado como institución, sí que cuestiona su comportamiento actual y una gran parte de sus reglas de juego, al ser incapaz de promover el bienestar general por centrarse en el interés individual y la acumulación de la riqueza en manos de unas pocas personas. Para garantizar el bien común, entendido éste como el interés general, hay que cambiar las reglas del mercado a través de la cooperación y la participación democrática (Campos Saavedra y Rodríguez Morilla, 2017; Gómez-Álvarez Díaz et al., 2017). Recientemente se han publicado algunos trabajos que analizan el modelo de la EBC desde la perspectiva de los recursos humanos (Ollé-Espluga et al., 2020; Aust et al., 2020).

Teniendo en cuenta lo apuntado, el objetivo de este trabajo consiste en analizar el modelo de la Economía del Bien Común (EBC) como un modelo apropiado para las empresas que buscan la sostenibilidad y que basan su comportamiento en la ética y la dimensión humana. Se describen sus principales características, así como su principal herramienta, el Balance del Bien Común, la cual permite cuantificar la aportación que las empresas pueden realizar al bien común a través de la creación de valor social y ambiental. Para determinar sus peculiaridades y diferencias respecto a otros modelos, se ha realizado un análisis comparativo con el enfoque de la Creación de Valor Compartido (CVC) de Porter y Kramer (2011) y con la Responsabilidad Social Corporativa (RSC).

\section{La Sostenibilidad Corporativa como punto de conexión entre la CVC y la EBC}

Para poder realizar un análisis comparativo entre el modelo de la EBC y los enfoques de la Creación de Valor Compartido (CVC) y de la Responsabilidad Social Corporativa (RSC), es necesario antes desarrollar los fundamentos teóricos de cada una de estas teorías. Por tanto, primero se analizará el enfoque de la CVC y su relación con la Sostenibilidad Corporativa (SC) y en segundo lugar se presentarán las bases del modelo de la EBC.

Porter y Kramer (2006) mantienen que la mayoría de las inversiones empresariales solo producen beneficios económicos para la empresa, careciendo de interés social, mientras que las contribuciones benéficas no relacionadas con el negocio solo generan beneficios sociales y ningún incremento del beneficio empresarial. Por el contrario, con la implementación de prácticas de RSE, las empresas consiguen lo que los autores denominan valor compartido, es decir, una combinación de valor económico y de valor social (Baron, 2008 y 2009). Las empresas que consiguen obtener valor compartido se denominan, según Porter y Kramer (2011), empresas 
híbridas, y solo este tipo de empresas son capaces, según estos autores, de conseguir la sostenibilidad, es decir, su supervivencia a largo plazo. Ni las empresas con ánimo de lucro (creadoras exclusivamente de valor económico), ni las empresas altruistas (creadoras exclusivamente de valor social) pueden ser sostenibles en el nuevo orden económico competitivo actual.

Se define el valor compartido como "... las políticas y las prácticas operacionales que mejoran la competitividad de una empresa a la vez que ayudan a mejorar las condiciones económicas y sociales en las comunidades donde opera" (Porter y Kramer, 2011:6). De esta definición se deduce que la empresa puede crear valor económico y, simultáneamente, crear también valor para la sociedad intentando dar solución a sus necesidades y retos; por ejemplo: el bienestar de los clientes, la sobreexplotación de los recursos naturales esenciales para el funcionamiento del negocio, la sostenibilidad de proveedores clave y/o la situación económica desfavorable de las comunidades en las que actúa la empresa, entre otros aspectos (Mohedano Suanes y Navarro Babiera, 2018).

En su descripción del concepto de valor compartido, Porter y Kramer (2011) señalan que las empresas han de ir más allá de la RSC, ya que estas políticas de responsabilidad social sitúan a la creación de valor social como algo periférico a la estrategia de la empresa y son utilizadas por las empresas como una forma de buscar legitimidad social e incrementar la cuenta de resultados a corto plazo (Porter y Kramer, 2006). Este enfoque coincide con la visión clásica de la RSC según la cual sus acciones contribuyen a mejorar la imagen y reputación de la empresa, entendidas por tanto como estrategias de marketing. Sin embargo, una estrategia basada en el valor compartido es una apuesta a largo plazo. Aunque sus resultados pueden requerir de más tiempo y mayor inversión inicial “...el retorno será un mayor valor económico y beneficios estratégicos más amplios para todos/as los/las participantes" (Porter y Kramer, 2011:4). Estamos, por tanto, ante un enfoque estratégico de la RSC, en el que las acciones de responsabilidad social no pueden ser entendidas como acciones puntuales y aisladas que la empresa va realizando en la medida en que las ve necesarias aplicar y que son implementadas dentro de un departamento o área concreta; sino que han de estar incluidas dentro de la estrategia de la empresa bajo un enfoque holístico (global e integrado). La integración de la RSC en la gestión estratégica ofrece un enfoque potencial que puede aumentar la capacidad de las empresas para afrontar el cambio hacia una gestión del medioambiente y de los aspectos sociales (Engert et al., 2016). De hecho, existen ya algunas investigaciones científicas que proponen una aproximación al proceso de integración de la RSC en la gestión estratégica, como por ejemplo sostienen Siebenhüner y Arnold (2007) al señalar que orientarse hacia la sostenibilidad implica utilizar tecnologías eficientes en el uso de los recursos, ofrecer productos y servicios que sean más ecológicos o introducir informes o memorias de sostenibilidad.

Bajo este enfoque o perspectiva, los planteamientos de la RSC quedarían relegados a un segundo plano, $\mathrm{o}$ al menos supeditados al enfoque de la sostenibilidad corporativa, un enfoque más moderno y avanzado que el de la RSC en el que se puede incorporar con total flexibilidad el concepto de la creación de valor compartido (Dyllick y Muff, 2016).

La Sostenibilidad Corporativa surge de la relación entre la Responsabilidad Social Empresarial (RSE) y el Desarrollo Sostenible (Henderson, 2007; Mirchandi e Ikerd, 2008) y el término 
se utiliza por primera vez en el Informe Brundtland (1987) de Naciones Unidas sobre el futuro del planeta y la relación entre medio ambiente y desarrollo, cuando se define como "las diversas formas de progreso que satisfacen las necesidades de la generación presente sin comprometer la capacidad de las generaciones futuras para satisfacer sus propias necesidades".

La Sostenibilidad Corporativa se entiende, según la mayor parte de sus definiciones (Salzman et al., 2005; Dyllick y Muff, 2016), como un enfoque de negocio inclusivo en el que se integran en su desarrollo los diferentes stakeholders de la empresa y cuyo fin es la creación de valor a largo plazo (Van Marrewijk, 2003; Montiel, 2008). La clave está, como ya se ha señalado, en que el valor creado sea tanto económico como social y ambiental (Dyllick y Hockerts, 2002) y beneficie, por tanto, al conjunto de los stakeholders (tanto internos como externos) y no solo a sus propietarios/as y directivos/as. Estamos, por tanto, ante un enfoque que se sustenta en la Teoría de los Stakeholders. Como veremos más adelante, el modelo de la EBC basa la medición de los impactos sociales y ambientales y de la contribución al bien común en la relación que la empresa mantiene con sus diferentes stakeholders (Mora Rodríguez et al., 2018). Sin embargo, al igual que el enfoque de la CVC de Porter y Kramer (2011), la Sostenibilidad Corporativa no tiene en cuenta el comportamiento ético de las empresas o lo deja relegado a un segundo plano, lo que impide que la empresa asuma sus responsabilidades y responda ante las expectativas legítimas generadas por los grupos de interés (Muñoz-Martín, 2013).

Por su parte, el modelo de la EBC se fundamenta en la necesidad que tienen las empresas de medir sus impactos sociales y ambientales, además de sus beneficios económicos y financieros (Felber, 2012; Campos Climent et al., 2020). Bajo este criterio, una empresa del bien común es aquella que es capaz de crear un triple valor: económico, social y ambiental; contribuyendo así a la sostenibilidad, tanto interna (de la propia empresa) como externa (de la sociedad en la cual lleva a cabo su actividad empresarial). Las empresas que utilizan este modelo se pueden definir, por tanto, como empresas sostenibles, de manera que las tres dimensiones de la sostenibilidad (económica, social y ambiental) se refuerzan mutuamente²: la dimensión económica favorece el desarrollo de las dimensiones social y ambiental y estas dos últimas dimensiones refuerzan la primera (Sanchis Palacio y Campos Climent, 2018). Solo así es posible un sistema económico y empresarial basado en los valores éticos y humanos, principios en los que se basa el modelo de la EBC, y se pueden alcanzar los objetivos del bien común, que son el bienestar social y la mejora de la calidad de vida, frente al ánimo de lucro y los intereses particulares, que son los principios que rigen el modelo del capitalismo neoliberal (Rodríguez Fernández, 2018).

Veamos a continuación cuáles son los valores que considera el modelo EBC para medir la contribución de una empresa al bien común, pues son estos valores la base de la medición del modelo y además se pueden encuadrar dentro de los Objetivos de Desarrollo Sostenible (ODS) de Naciones Unidas, los que explican la relevancia de este modelo en relación con la sostenibilidad corporativa (Campos Climent et al., 2019). El modelo contempla cuatro tipos de valores diferentes: 1) la dignidad humana, 2) la solidaridad y justicia social, 3) la sostenibili-

2. Más adelante se describirán cuáles son los valores en los que se sustenta el modelo de la EBC para garantizar la sostenibilidad corporativa. 
dad ambiental o ecológica y 4) la transparencia y codecisión o coparticipación (Felber, 2012; Rodríguez Fernández, 2018). Estos cuatro grupos de valores están relacionados con la ética y el humanismo, de manera que las empresas que contribuyen a dichos valores se enmarcan dentro de lo que podemos denominar empresas sostenibles o del bien común.

El trabajo de Dyllick y Muff (2016) establece una tipología de la Sostenibilidad Corporativa basada en 4 fases o etapas diferentes: negocios usuales, SC 1.0, SC 2.0 y SC 3.0. La SC 3.0, que es la etapa más avanzada del proceso, se caracteriza por la creación de valor para el bien común, por lo que el modelo de la EBC estaría incluido dentro de esta etapa más avanzada de la SC; así lo señalan los autores en el trabajo (Dyllick y Muff, 2016: 169).

\section{El modelo organizativo de la Economía del Bien Común}

El modelo de la EBC, desde el punto de vista organizativo, ofrece un instrumento de medición del valor social y ambiental que le sirve a la empresa como complemento de la medición de su valor económico y financiero, lo que lo convierte en un modelo con una visión global, integrada y flexible ${ }^{3}$. Este instrumento es el Balance del Bien Común (BBC), que a diferencia de otros instrumentos, se puede aplicar a cualquier tipo de organización: administraciones y empresas públicas, empresas privadas con ánimo de lucro y empresas privadas sin ánimo de lucro ${ }^{4}$; e incluso también a comunidades y personas (Campos Climent et al., 2019). El BBC, no solo sirve para medir los impactos sociales y ambientales de una organización, sino que también es útil para la mejora de la gestión en estos aspectos, ya que incorpora un plan de mejora continua mediante el cual la organización introduce medidas dirigidas a mejorar su impacto en los aspectos sociales y medioambientales donde se han detectado deficiencias o insuficiencias (Felber et al., 2019).

El plan de mejora continua de la EBC incluye un análisis de la situación, un diagnóstico y valoración y la elaboración de un informe final, y puede ser auditado, bien mediante el sistema peer (revisión por pares) o bien mediante auditoría externa. Este plan de mejora se ha de integrar en el plan estratégico o en el plan de acción de la empresa y deberá incluir (Amigo Pérez y Ribera Esteve, 2018): fijación de objetivos claros, determinación de las acciones necesarias para alcanzar los objetivos previstos, establecimiento de los indicadores que habrá que mejorar, plazos previstos para la consecución de los objetivos, responsables de la aplicación de cada acción y personas y recursos (tiempo, recursos materiales y financiación) destinados

3. Se puede considerar como herramienta más antigua relacionada con el modelo EBC, la Triple Bottom Line (Carroll 1999). Otras herramientas más actuales son el Canvas de triple capa (Joyce, Paquin y Pigneur, 2015) y el Balance Scorecard de cinco dimensiones (Figge et al., 2002; Lusk et al., 2006).

4. La BCorporation, por ejemplo, que también es un instrumento alternativo relativamente reciente para las empresas sostenibles, solamente se aplica a las empresas privadas con ánimo de lucro, excluyendo a las entidades no lucrativas y a las entidades y corporaciones públicas (Sanchis Palacio y Amigo Pérez, 2018). 
a cada acción. Esto supone un plus en relación con las memorias de sostenibilidad y con otras herramientas que se utilizan en la actualidad por las empresas, que le proporciona un valor añadido a tener en cuenta (Campos Climent et al., 2019).

En este sentido, podemos decir que el BBC no solo es un indicador o instrumento de medida, sino que también es una herramienta estratégica que sirve para facilitar el cambio organizativo, dirigiendo la empresa hacia posiciones más sostenibles y éticas. Las ventajas que le puede reportar a la empresa son (Amigo Pérez y Ribera Esteve, 2018): 1) diferenciación: se pueden indentificar como empresas éticas y sostenibles; 2) compromiso: el éxito está basado en el compromiso de las personas que forman el equipo de trabajo; 3): innovación: se consigue innovación social, con mayores posibilidades de réplica y extensión del modelo; y 4) acceso a la inversión: mediante criterios basados en la inversión socialmente responsable.

Las empresas del bien común pueden obtener una certificación o etiqueta propia, que depende de la obtención de tres tipos de "semillas" diferentes según el estado del proceso de verificación del modelo: 1 semilla: implementación del balance a nivel interno sin ser auditado; 2 semillas: el balance es auditado mediante el sistema peer o revisión por pares en el que participan como mínimo tres empresas coordinadas por un/a consultor/a certificado/a; 3 semillas: el balance es auditado por un/a auditor/a externo/a. La etiqueta se obtiene cuando se consiguen las 3 semillas (Amigo Pérez y Ribera Esteve, 2018).

La Matriz del Bien Común (MBC) es el instrumento que se utiliza en la aplicación del BBC para poder cuantificar la medición de las diferentes variables que lo conforman y que quedan recogidas en 20 "temas" diferentes, que son el resultado de cruzar en la MBC los cuatro valores del bien común (dignidad humana, justicia y solidaridad, sostenibilidad ambiental y transparencia y participación democrática) con los cinco grupos de contacto o de interés (los/as proveedores/as, los/as propietarios/as y financiadores/as, las personas empleadas, los/las clientes/as y otras empresas y el entorno social) (Felber et al., 2019). Miden aspectos tan diversos como "la utilidad de los productos/servicios que ofrecen las empresas, cómo son las condiciones laborales en una empresa, si se produce o no ecológicamente, cómo se reparten los ingresos, si las decisiones en una empresa se toman de manera democrática, la existencia o no de diferencias en las remuneraciones entre hombres y mujeres, etc." (Rodríguez Fernández, 2018:12).

A cada uno de los 20 temas se le asigna una puntuación máxima de 50 puntos; así pues, la puntuación máxima que puede obtener una empresa que aplica la MBC es de 1.000 puntos. También se aplican aspectos negativos (uso de productos contaminantes en exceso, condiciones de abuso sobre los proveedores, sobre los clientes o sobre otras empresas, ...), que restan puntuación, pudiendo ser esta de hasta -3.600 puntos. También se ha de tener en cuenta que las variables se pueden ponderar en función de determinados factores como son el tamaño de la organización, los movimientos financieros con propietarios/as, proveedores/as y personas empleadas, los riesgos de impacto social negativo en los países de los principales proveedores y sector de la actividad y los riesgos de impactos medioambientales y sociales negativos asociados $^{5}$. Una empresa del bien común se puede clasificar en cuatro clases diferentes según la

5. Para conocer con mayor detalle los temas y aspectos considerados en la MBC se puede consultar Amigo Pérez y Ribera Esteve (2018) y el enlace https://economiadelbiencomun.org/primeros-pasos/ 
puntuación obtenida: empresa principiante (entre 1 y 100 puntos), empresa avanzada (entre 101 y 300 puntos), empresa experimentada (entre 301 y 600 puntos) y empresa ejemplar (más de 600 puntos). Un estudio empírico realizado a nivel europeo, demuestra que las empresas que utilizan la MBC, y que son aproximadamente unas 400 en toda Europa (centradas en Austria, Alemania, Suiza, España e Italia principalmente), se sitúan en un nivel avanzado (69\% del total) con una puntuación media de 497 puntos (Campos Climent et al., 2019). La estructura de la MBC queda recogida en la Figura 1.

\section{Figura 1. Matriz del Bien Común 5.0}

\section{MATRIZ DEL BIEN COMÚN 5.0}

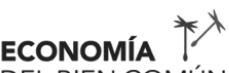

DEL BIEN COMÚN

\begin{tabular}{|c|c|c|c|c|}
\hline GRUPO DE INTERÉS & $\begin{array}{l}\text { DIGNIDAD } \\
\text { HUMANA }\end{array}$ & $\begin{array}{l}\text { SOLIDARIDAD Y } \\
\text { JUSTICIA }\end{array}$ & $\begin{array}{l}\text { SOSTENIBILIDAD } \\
\text { MEDIOAMBIENTAL }\end{array}$ & $\begin{array}{c}\text { TRANSPARENCIA Y } \\
\text { PARTICIPACIÓN } \\
\text { DEMOCRÁTICA }\end{array}$ \\
\hline $\begin{array}{l}\text { A: } \\
\text { PROVEEDORES }\end{array}$ & $\begin{array}{l}\text { A1 Dignidad humana en } \\
\text { la cadena de suministro }\end{array}$ & $\begin{array}{l}\text { A2 Justicia y solidaridad } \\
\text { en la cadena de } \\
\text { suministro }\end{array}$ & $\begin{array}{l}\text { A3 Sostenibilidad } \\
\text { medioambiental en la } \\
\text { cadena de suministro }\end{array}$ & $\begin{array}{l}\text { A4 Transparencia y } \\
\text { participación } \\
\text { democrática en la } \\
\text { cadena de suministro }\end{array}$ \\
\hline $\begin{array}{l}\text { B: } \\
\text { PROPIETARIOS Y } \\
\text { PROVEEDORES } \\
\text { FINANCIEROS }\end{array}$ & $\begin{array}{l}\text { B1 Actitud ética en la } \\
\text { gestión de recursos } \\
\text { financieros }\end{array}$ & $\begin{array}{l}\text { B2 Actitud solidaria en } \\
\text { la gestión de recursos } \\
\text { financieros }\end{array}$ & $\begin{array}{l}\text { B3 Inversiones } \\
\text { financieras sostenibles y } \\
\text { uso de los recursos } \\
\text { financieros }\end{array}$ & $\begin{array}{l}\text { B4 Propiedad y } \\
\text { participación } \\
\text { democrática }\end{array}$ \\
\hline $\begin{array}{l}\text { C: } \\
\text { PERSONAS } \\
\text { EMPLEADAS }\end{array}$ & $\begin{array}{l}\text { C1 Dignidad humana en } \\
\text { el puesto de trabajo }\end{array}$ & $\begin{array}{l}\text { C2 Formalidad de los } \\
\text { contratos de trabajo }\end{array}$ & $\begin{array}{l}\text { C3 Promoción de la } \\
\text { responsabilidad } \\
\text { medioambiental de los } \\
\text { trabajadores }\end{array}$ & $\begin{array}{l}\text { C4 Transparencia y } \\
\text { participación } \\
\text { democrátia interna }\end{array}$ \\
\hline $\begin{array}{l}\text { D: } \\
\text { CLIENTES Y OTRAS } \\
\text { ORGANIZACIONES }\end{array}$ & $\begin{array}{l}\text { D1 Actitud ética con los } \\
\text { clientes }\end{array}$ & $\begin{array}{l}\text { D2 Cooperación y } \\
\text { solidaridad on otras } \\
\text { empresas }\end{array}$ & $\begin{array}{l}\text { D3 Impacto ambiental } \\
\text { del uso y de la gestión } \\
\text { de residuos de los } \\
\text { productos y servicios }\end{array}$ & $\begin{array}{l}\text { D4 Participación de los } \\
\text { clientes y transparencia } \\
\text { de producto }\end{array}$ \\
\hline $\begin{array}{l}\text { E: } \\
\text { ENTORNO SOCIAL }\end{array}$ & $\begin{array}{l}\text { E1 Propósito e impacto } \\
\text { positivo de los } \\
\text { prodcutos y servicios }\end{array}$ & $\begin{array}{l}\text { E2 Contribución a la } \\
\text { comunidad }\end{array}$ & $\begin{array}{l}\text { E3 Reducción del } \\
\text { impacto } \\
\text { medioambiental }\end{array}$ & $\begin{array}{l}\text { E4 Transparencia y } \\
\text { participación } \\
\text { democrática del } \\
\text { entorno social }\end{array}$ \\
\hline
\end{tabular}

Fuente: Asociación Federal Española de la EBC.

El desempeño de la MBC es doble: por una parte, es un instrumento de medición del bien común por parte de la empresa; y por otra, es un instrumento estratégico que alinea los valores de la EBC con los grupos de interés de la empresa. Su consideración como un instrumento de medición le otorga un valor destacado, ya que permite comparar el modelo de la EBC con otros tipos de modelos de sostenibilidad como los que aporta el GRI (Global Reporting Initiative) o las memorias de sostenibilidad, por ejemplo. Así, es importante destacar que ya existe un estudio (Felber et. al, 2019) que demuestra la validez estadística de la MBC, al señalar que una 
parte importante de sus indicadores de sostenibilidad, sobre todo los que hacen referencia al valor social, son apropiados y válidos; si bien, se demuestra también algunas limitaciones del modelo que conviene conocer y que afectan a la relación de los valores del bien común con los clientes y con el entorno social.

De lo analizado, se puede señalar que la MBC no es solo un instrumento de medición, si no que también posee un carácter estratégico, al igual que otras matrices utilizadas en el campo del management (Matriz de crecimiento de Ansof, Matriz DAFO, ...). La MBC relaciona los valores del bien común con los diferentes stakeholders, lo que permite establecer estrategias a seguir por parte de las empresas. Sitúa las relaciones entre la empresa y sus stakeholders (internos y externos) en un contexto estratégico, definiendo acciones a llevar a cabo. Estas acciones estratégicas tienen como finalidad incrementar la contribución al bien común por parte de la empresa desde una perspectiva a largo plazo. Lo que importa no es la puntuación que una empresa puede obtener en un momento determinado del tiempo (resultado de aplicar la $\mathrm{MBC}$ ), si no su evolución a lo largo del tiempo, de manera que a través de la implantación de un plan de mejora basado en el informe del bien común, puede ir incrementando sus niveles de creación de valor social y ambiental en la medida en que va desarrollando nuevas acciones.

\section{Análisis comparativo entre la CVC y la EBC desde la perspectiva de la SC}

Se deduce de todo lo anterior que tanto el modelo de la EBC como el enfoque de la CVC destacan que las empresas sostenibles (denominadas empresas del bien común en el caso de la EBC y empresas híbridas en el caso de la CVC) son aquellas que son capaces de crear el triple valor de la sostenibilidad corporativa: económico, social y ambiental. En otros términos, se puede destacar que la sostenibilidad corporativa se basa en la interrelación entre el valor económico (económico-financiero diría Felber) y los valores social y ambiental ${ }^{6}$, de manera que los tres valores se refuerzan mutuamente. Así, crear valor social (y ambiental) permite también incrementar el valor económico de la empresa, lo que contrasta con lo señalado por las posiciones opuestas a la responsabilidad social (políticas monetaristas de Milton Friedman), incluso con algunas posturas enmarcadas dentro de la RSC que señalan que las acciones de responsabilidad social obligan a sacrificar parte de los beneficios económicos y financieros de las empresas a cambio de la mejora de su imagen y reputación (Baron, 2009).

Frente a las posiciones que indican que aplicar la RSC tiene un coste económico para la empresa, la CVC y el modelo de la EBC coinciden en señalar que crear valor social y ambiental puede ser también beneficioso para la empresa, por lo que las acciones de responsabilidad social han de ser entendidas, no como un coste, sino como una inversión real que a largo plazo

6. Porter y Kramer (2011) hacen referencia solamente al valor social, dentro del cual incluyen también el valor ambiental o ecológico. 
puede repercutir positivamente en los resultados financieros de la empresa. Este aspecto es determinante para comprender el verdadero alcance de los planteamientos que se vierten en los nuevos enfoques de la sostenibilidad corporativa, y es el que sirve como punto de referencia para ofrecer una visión de la empresa más avanzada a la de la RSC. Ahora bien, el modelo de la EBC destaca que no es suficiente con la ciudadanía corporativa (persona consumidora responsable) y la empresa sostenible, ya que el sistema capitalista está pensado y enfocado en la maximización del beneficio económico y el egoísmo; por tanto, se hace necesario una reforma del comportamiento de los mercados a través de las Administraciones Públicas, que son las que han de motivar este tipo de comportamientos mediante incentivos fiscales, introducción de clausulas sociales en las contrataciones públicas y ayudas económicas, entre otro tipo de políticas públicas (Gómez Calvo y Gómez-Álvarez Díaz, 2016; Gómez-Álvarez Díaz et al., 2017).

En este sentido, tanto el enfoque de la CVC como el modelo de la EBC le confieren un papel clave a la transparencia en los mercados y a la cooperación, entendidos ambos como conceptos determinantes de la sostenibilidad corporativa y como condiciones necesarias para crear valor compartido (Beschorner, 2014). El modelo de la EBC propone sustituir la competencia por la cooperación, por lo que este último concepto adquiere un papel determinante; y el enfoque de la CVC, aunque mantiene la necesidad de la competencia como clave en el funcionamiento de las empresas, también le otorga a la cooperación una función relevante en el proceso de creación de valor por parte de las empresas. En todo caso, competencia y cooperación no son prácticas incompatibles, sino que incluso se pueden complementar ${ }^{7}$, pues se pueden dar acuerdos de cooperación entre empresas competidoras o entre empresas que llevan a cabo actividades diferentes dentro del sistema de valor. En todos estos casos, la confianza y el respeto mutuo son características que garantizan el éxito de la cooperación y que ofrecen una visión más ética y humanista del comportamiento empresarial.

El modelo de la EBC va más allá del enfoque de la CVC, pues contempla la cooperación como un elemento fundamental para su implementación, tanto a nivel interno como externo, y plantea que ésta ha de sustituir a la competencia. En cambio, el enfoque de la CVC introduce la relevancia de la cooperación a través del concepto de clusters locales, partiendo de la idea de que ninguna empresa es autosuficiente, de manera que la productividad y la innovación están altamente influidas por las relaciones entre las diferentes empresas, las infraestructuras u otros agentes sociales e institucionales que intervienen en el proceso (Porter y Kramer, 2011). Sin embargo, en ningún caso el enfoque de la CVC plantea sustituir la cooperación por la competencia, sino que ésta última sigue siendo la clave del éxito empresarial. Se encuentra aquí una diferencia significativa entre el modelo de la EBC y el enfoque de la CVC, que conviene señalar y analizar.

A diferencia del modelo de la EBC, el enfoque de la CVC no plantea reemplazar la competencia por la cooperación, pues en el centro de la misma definición que aportan de valor com-

7. Pudiendo ser analizadas desde la perspectiva de la coo-petición (o coo-petencia), un concepto que es introducido en 1996 por Adam M. Brandenburger y Barry J. Nalebuff (Nalebuffy Brandenburger, 1996). Según este enfoque, se entiende la cooperación como un estado natural de los seres humanos que contribuye a la armonía económica, política y social. 
partido, Porter y Kramer (2011) sitúan a la competitividad como un elemento principal. La creación de valor compartido la entienden como el instrumento para conseguir el fin principal de la empresa que es la obtención de una ventaja competitiva sostenible; de manera que las prácticas de RSC contribuyen al incremento de la productividad de la empresa. Por tanto, en el enfoque de la CVC, el objetivo principal es la capacidad competitiva de la empresa, y la creación de valor social (y ambiental) se convierten en el medio o el instrumento para alcanzar dicho fin. Es decir, valor económico y valor social están en un mismo nivel, de manera que para Porter y Kramer (2011), el crecimiento económico y empresarial puede ser un objetivo perfectamente legítimo. Sin embargo, en el modelo de la EBC de Felber (2012), el beneficio económico y financiero ha de ser el medio o instrumento para contribuir al bien común y no un fin en sí mismo. De la misma manera, el crecimiento económico y empresarial no puede ser nunca entendido como un fin u objetivo, sino como el instrumento para alcanzar el bienestar social y la mejora de la calidad de vida.

Por último, la CVC no incluye el valor ético como un aspecto clave que han de considerar las empresas en su comportamiento con la sociedad, sino que éste es considerado como suplementario. Por este motivo, la empresa puede crear valor social pero no garantiza su legitimidad, pues no se puede asegurar que asuma la responsabilidad de sus actos, tal como señalan autores como Muñoz-Martín (2013), Hartman y Werhane (2013) y Crane et al. (2014). Por lo tanto, se puede decir que el modelo de la EBC es un modelo más completo que la CVC y la RSC, pues a través del desarrollo de sus valores éticos y sociales, consigue humanizar las empresas y la economía.

De todo lo analizado, se deduce que existen diferencias significativas entre los modelos de la CVC y de la EBC, tal y como se resume y se puede observar en la Tabla 1.

\section{Tabla 1. Principales diferencias entre los modelos de la CVC y la EBC}

\begin{tabular}{l|ll} 
Concepto & CVC & EBC \\
\hline Objetivos & Beneficio económico y crecimiento & Bien común y bienestar social \\
\hline Agentes intervinientes & Empresa y Sociedad & Empresa, Sociedad y AA.PP. \\
\hline Mercado & Reformarlo & Transformación profunda \\
\hline Cooperación & Instrumento al servicio de la competencia & Sustituir a la competencia \\
\hline Valores éticos & No se incluyen & Sí se incluyen \\
\hline
\end{tabular}

Fuente: Elaboración propia.

Por tanto, podemos identificar el modelo de la EBC como un modelo más próximo al concepto de sostenibilidad corporativa que los enfoques de la CVC y de la RSC. Si definimos el concepto de sostenibilidad corporativa como la garantía de la supervivencia a largo plazo de las generaciones futuras mediante la conservación del medio ambiente y de la recuperación de los valores sociales y humanos, podemos afirmar que el modelo de la EBC es sin duda un 
modelo holístico e integrado frente a las limitaciones de los otros enfoques y, por tanto, puede contribuir con mayor garantía al éxito de la sostenibilidad corporativa.

No obstante, las aportaciones del enfoque de la CVC de Porter y Kramer (2011) a cómo crear valor compartido, facilita la implantación del modelo de la EBC, pues como señalan Mohedano Suanes y Navarro Babiera (2018), algunas de las acciones que permiten crear valor compartido son también una forma de incorporar a la actuación de la empresa los valores de la EBC. Así por ejemplo, tomar medidas destinadas a conseguir la igualdad de género con el fin de incrementar las competencias de la empresa (desde el enfoque de la CVC se conseguirá un incremento de la productividad) puede ser una acción que a su vez contribuye a la mejora del valor de la EBC de la dignidad humana (igualdad entre hombres y mujeres). También establecer acuerdos de cooperación con proveedores/as locales con el fin de mejorar las competencias tanto de éstos/estas como de la propia empresa (según el enfoque de la CVC mejorará su eficiencia a través de la mejora de la calidad de sus productos), es una medida que contribuye directamente al valor de la solidaridad (recogido dentro del modelo de la EBC). Tomar medidas dirigidas a reducir los efectos contaminantes introduciendo el uso de energías limpias o renovables produce un doble efecto positivo: para la empresa porque le permite reducir costes energéticos (aumento de la productividad según la CVC) y para la sociedad, contribuyendo así a conseguir el valor de la EBC de la sostenibilidad ecológica. Destinar parte de los beneficios económicos de la empresa a acciones educativas y culturales, contribuye a mejorar el valor de la justicia social (incluido dentro del modelo EBC), pero también puede servir para que la empresa pueda disponer de personal con un mayor grado de cualificación (incrementando su productividad según la CVC). Por último, crear redes locales con otras empresas y con clientes / as y proveedores/as favorece la participación y la toma de decisiones compartidas, de manera que la empresa consigue recursos y capacidades que por ella solo sería incapaz de alcanzar (criterio CVC) y a la vez se consigue una mejora de la transparencia y la participación democrática, que son valores recogidos en el modelo de la EBC.

\section{Discusión y conclusiones}

La responsabilidad social no es un instrumento suficiente para favorecer el cambio de modelo económico y productivo. Su aplicación se ha centrado en la mejora de la reputación y la imagen de la empresa, de manera que básicamente es una estrategia de marketing. En este sentido, se hace necesario un enfoque de la empresa en el que la responsabilidad social forme parte del corazón de la estrategia de la empresa y no se trate solo de acciones aisladas y concretas. Ahora bien, el modelo de la EBC, al igual que otros modelos alternativos, se puede convertir también en una herramienta de marketing según el uso que se le dé. En este sentido, analizar los factores motivadores de las empresas del bien común para la implantación de este modelo puede ser un aspecto relevante a investigar, como ya adelantan Campos Climent et al. (2019) en estudios empíricos realizados sobre la aplicación del modelo en Europa. 
Durante las últimas décadas, han surgido nuevos enfoques empresariales que ponen el énfasis en la sostenibilidad, tratando de garantizar la supervivencia de la empresa a largo plazo. Dentro de estos enfoques, destaca el de la creación de valor compartido de Porter y Kramer (2011), cuya aportación supone un antes y un después en el camino hacia la sostenibilidad corporativa. El valor económico y el valor social y ambiental de una empresa no son contradictorios (para conseguir uno hay que sacrificar parte del otro), sino que se refuerzan mutuamente (al conseguir uno, se incrementa también el otro). Este planteamiento supone dar un paso más allá de la responsabilidad social, al situar este tipo de acciones de la empresa dentro de su estrategia, aportando una visión a largo plazo.

Sin embargo, estos nuevos enfoques de la sostenibilidad, aunque relacionan el valor económico con el valor social y ambiental de la empresa, no tienen en consideración valores tan importantes como la ética y el humanismo, de manera que siguen priorizando la maximización del beneficio y la competencia sobre el bien común y la cooperación, convirtiendo el crecimiento y el aumento de tamaño en fines a alcanzar. El modelo de la Economía del Bien Común relega el crecimiento y el aumento de tamaño a instrumentos o medios, de manera que el objetivo de la economía ha de ser la mejora de calidad de vida de las personas y el bienestar de la sociedad. También considera los valores éticos y humanos como determinantes en el funcionamiento de la economía y las empresas.

Se trata de un modelo que se puede aplicar no solo en las empresas sociales (empresas de economía social y solidaria y cooperativas, entre otras), sino también en cualquier tipo de empresa, sea pública o privada (Gómez Calvo y Gómez-Alvarez Díaz, 2016). En este sentido, la aportación del modelo es significativa, pues propone ampliar el valor social de las empresas también a las empresas de capitales. Se trata de extender un modelo que complementa las ganancias con los fines sociales a cualquier tipo de organización, independientemente de su forma jurídica, tamaño o estructura de la propiedad. Representa pues un enfoque de innovación social a través del cual cambiar la forma de entender las relaciones y el comportamiento de las organizaciones.

Pero el modelo de la Economía del Bien Común no es solo un instrumento para medir el valor social y ambiental de la empresa, sino que además es un proceso de mejora de la gestión de las organizaciones. A través del Balance del Bien Común, la empresa consigue cuantificar su aportación al bien común (sus impactos sociales y ambientales positivos), pero también puede diseñar un sistema de mejora continua, mediante el cual ir incrementando sus aportaciones a la sociedad, a la vez que se consigue un modelo de gestión más eficiente y eficaz, garantizando su supervivencia a largo plazo. Por eso decimos que el modelo de la Economía del Bien Común es el camino hacia la sostenibilidad corporativa, porque a través de sus instrumentos las empresas consiguen mejorar de forma continua su capacidad de gestión desde una posición ética y humanista.

El Balance del Bien Común permite cuantificar todo tipo de variables sociales y ambientales, de manera que el modelo incluye dentro de su funcionamiento enfoques tan diversos como la economía feminista, la economía circular, la economía ecológica o la economía democrática entre otros: plantea medidas para alcanzar la igualdad de género en las empresas a través de 
salarios y condiciones laborales iguales entre hombres y mujeres y a través del acceso de las mujeres a los puestos directivos; propone, no solo el uso de energías renovables que reduzcan impactos negativos ambientales, sino también la gestión eficiente de los recursos a través de la reducción del consumo y la reutilización de los mismos; y propone medidas para la transparencia y la participación democrática directa en las decisiones de las empresas. También conecta con la Economía social, solidaria y cooperativa, pues considera este tipo de organizaciones como empresas ejemplares del bien común, por su filosofía y principios.

Se puede afirmar, por tanto, que se trata de un modelo empresarial de carácter holístico (global e integrado) en el que el fin es la sostenibilidad. A diferencia de otros enfoques y modelos como el de la Creación de Valor Compartido o el de la Responsabilidad Social Corporativa, posee unas características que lo hacen especialmente adecuado para la búsqueda de la sostenibilidad corporativa: 1) es aplicable a cualquier tipo de organización, por lo que se puede decir que es un modelo empresarial de ámbito universal; 2) sirve para medir cualquier tipo de variable social y ambiental, cuantificándolas mediante unas puntuaciones, por lo que es un modelo mesurable; 3) sus variables e indicadores se ponderan en función de determinados factores de contingencia de la organización, por lo que se puede afirmar que se trata de un modelo flexible; y 4) permite introducir medidas de mejora continua desde una perspectiva estratégica, por lo que se puede decir que es un modelo dinámico.

Aunque se trata de un modelo joven, de apenas 10 años de existencia, son ya cientos las empresas que lo están aplicando, principalmente en el centro y sur de Europa, y su ámbito de actuación va ampliándose a empresas privadas de todas las características, entidades no lucrativas, municipios, universidades y comunidades de personas. Habrá que analizar cuál es la evolución y el grado de implantación que el modelo de la EBC es capaz de conseguir en un futuro próximo.

\section{Referencias bibliográficas}

AMIGO PÉREZ, M. \& RIBERA ESTEVE, M. (2018): “El Balance del Bien Común”, En: Sanchis Palacio, J.R. \& Amigo Pérez, M. (Coords.), El modelo de la Economía del Bien Común. Aplicación en la empresa/organización y casos prácticos, 121-140. Delta Publicaciones, Madrid.

AUST, I., MATTHEWS, B. \& MULLER-CAMEN, M. (2020): “Common Good HRM: A Paradigm Shift in Sustainable HRM?", Human Resource Management Review, 30(3), 100705. D0I: 10.1016/j. hrmr.2019.100705.

BARON, D. (2008): "Managerial contracting and corporate social responsibility", Journal of Public Economics, 92, 268-288. D0I: 10.1016/j.jpubeco.2007.05.008

BARON, D. (2009): "A positive theory of moral management, social pressure, and corporate social performance", Journal of Economics and Management Strategy, 18, 7-43.

DOI: 10.1111/j.1530-9134.2009.00206.x 
BESCHORNER, T. (2014): "Creating shared value: The one-trick pony approach", Business Ethics Journal Review, 1(17), 106-112. D0I: 10.12747/bejr2013.01.17.

BRUNDTLAND, G.H. (1987): Informe de la Comisión Mundial sobre el Medio Ambiente y el Desarrollo: Nuestro Futuro Común, Comisión Brundtland, New York.

CAMPOS CLIMENT, V., SANCHIS PALACIO, J.R. \& EJARQUE CATALÁ, A.T. (2019): "El modelo de la Economía del Bien Común. Un estudio empírico sobre su aplicación a la empresa privada”, REVESCO, Revista de Estudios Cooperativos, 132, 46-76, DOI: 10.5209/REVE.64304.

CAMPOS CLIMENT, V., SANCHIS PALACIO, J.R. \& EJARQUE CATALÁ, A.T. (2020): "Social entrepreneurship and Economy for the Common Good: Study of their relationship through a bibliometric analysis", The International Journal of Entrepreneurship and Innovation, 21(3), 156-167. DOI: $10.1177 / 1465750319879632$.

CAMPOS SAAVEDRA, L.F. \& RODRÍGUEZ MORILLA, C. (2017): "Los «Bienes Democráticos» en la Economía del Bien Común: cuatro vías para su delimitación conceptual", CIRIEC-España, Revista de Economía Pública, Social y Cooperativa, 90, 223-252. DOI: 10.7203/CIRIEC-E.90.9087.

CARROLL, A.B. (1999): "Corporate Social Responsibility: Evolution of a Definitional Construct”, Business \& society, 38(3), 268-295. D0I: 10.1177/000765039903800303.

CHAVES ÁVILA, R. \& MONZÓN CAMPOS, J.L. (2018): “La economía social ante los paradigmas económicos emergentes: innovación social, economía colaborativa, economía circular, responsabilidad social empresarial, economía del bien común, empresa social y economía solidaria", CIRIEC-España, Revista de Economía Pública, Social y Cooperativa, 93, 5-50, D0I: 10.7203/CIRIEC-E.93.12901.

CHOMSKY, N. (2002): El bien común. Entrevistas por David Barsamian, Siglo XXI Editores, Madrid.

COMITÉ ECONÓMICO Y SOCIAL EUROPEO [CESE] (2016): Dictamen del Comité Económico y Social Europeo sobre el tema "La Economía del Bien Común: un modelo económico sostenible orientado a la cohesión social", Dictamen EC0/378 -EESC- 2015-02060-00-00-AC-TRA (ES) 1/113, documento consultado en:

http://eur-lex.europa.eu/legal-content/ES/TXT/PDF/?uri=CELEX:52015IE2060\&from=ES.

CRANE, A., PALAZZO, G., SPENCE, L.J. \& MATTEN, D. (2014): "Contesting the value of "creating shared value", California Management Review, 56(2), 130-153. DOI: 10.1525/ CMR.2014.56.2.130.

DYLLICK, T. \& HOCKERTS, K. (2002): "Beyond the business case for corporate sustainability", Business Strategy and the Environment, 11(2), 130-141. D0I: 10.1002/bse.323. 
DYLLICK, T. \& MUFF, K. (2016): “Clarifyng the meaning of sustainable business: introducing a tylopogy from business-as-usual to true business sustainability", Organization \& Environment, 29(2), 156-174. DOI: 10.1177/1086026615575176.

ENGERT, S., RAUTER, R. \& BAUMGARTNER, R.J. (2016): "Exploring the integration of corporate sustainability into strategic management: a literature review", Journal of Cleaner Production, 112, 2833-2850, DOI: 10.1016/J.JCLEPR0.2015.08.031.

ENTRENA JIMÉNEZ, S. \& MARTÍNEZ RODRÍGUEZ, F.M. (2013): "Educación y cambio social: del capitalismo neoliberal a la economía del bien común", En: Torío López, S., García-Pérez, 0., Peña Calvo, J.V. \& Fernández García, C.M. (Coords.), Crisis social y Estado del Bienestar: las respuestas de la pedagogía social, 72-77. Servicio de Publicaciones de la Universidad de Oviedo, Oviedo. https://dialnet.unirioja.es/servlet/articulo?codigo=4481464.

FELBER, C. (2008): Nuevos valores para la economía. Una alternativa al comunismo y al capitalismo, Franz Deuticke, Leipzig.

FELBER, C. (2012): La economía del bien común, Ediciones Deusto, Barcelona.

FELBER, C., CAMPOS CLIMENT, V., \& SANCHIS PALACIO, J.R. (2019): "The Common Good Balance Sheet, an Adequate Tool to Capture Non-Financials?", Sustainability, 11(14), 3791, D0I: $10.3390 /$ su11143791.

FIGGE, H.T., HAHN, T., SCHALTEGGER, S. \& WAGNER, M. (2002): "The Sustainability Balanced Sorecard - Linking sustainability management to busness strategy", Business Strategy and the Environment, 11(5), 269-284. D0I: 10.1002/bse.339.

GÓMEZ-ÁLVAREZ DÍAZ, R., MORALES SÁNCHEZ, R. \& RODRÍGUEZ MORILLA, C. (2017): “La Economía del Bien Común en el ámbito local", CIRIEC-España, Revista de Economía Pública, Social y Cooperativa, 90, 189-222. DOI: 10.7203/CIRIEC-E.90.8898.

GÓMEZ CALVO, V. \& GÓMEZ-ÁLVAREZ DÍAZ, R. (2016): “La economía del bien común y la economía social y solidaria, ¿son teorías complementarias?", CIRIEC-España, Revista de Economía Pública, Social y Cooperativa, 87, 257-294. DOI: 10.7203/CIRIEC-E.87.8807.

HARTMAN, L.P. \& WERHANE, P.H. (2013): The Global Corporation: Sustainable, Effective and Ethical Practices, A Case Book, Routledge, Londres.

HENDERSON, J.C. (2007): "Corporate social responsibility and tourism: Hotel companies in Phuket, Thailand, after the Indian Ocean tsunami", International Journal of Hospitality Management, 26(1), 228-239. DOI: 10.1016/j.ijhm.2006.02.001.

JACKSON, T. (2011): Prosperity without Growth: Economics for a Finite Planet, Routledge, Londres. 
JOYCE, A., PAQUIN, R.L. \& PIGNEUR, Y. (mayo 2015): The triple layered business model canvas: a tool to design more sustainable business models [Comunicación en congreso]. ARTEM Organizational Creativity International Conference. Nancy, France.

KRUGMAN, P. (2012): ;Acabad ya con esta crisis!, Editorial Crítica, Barcelona.

LUSK, E.J., HALPERIN, M. \& ZHANG, B.D. (2006): "The Balanced Scorecard: Suggestions for Rebalancing", Problems and Perspectives in Management, 4(2), 100-114.

MICHELINI, L. \& FIORENTINO, D. (2012): "New business models for creating shared value", Social Responsibility Journal, 8(4), 561-577. DOI: 10.1108/17471111211272129.

MIRCHANDI, D. \& IKERD, J. (2008): “Building and maintaining sustainable organizations", Organization Management Journal, 5(1), 40-51. D0I: 10.1057/omj.2008.6.

MOHEDANO SUANES, A. \& NAVARRO BABIERA, C. (2018): "Teorías y enfoques organizativos que sustentan el modelo de la Economía del Bien Común", En: Sanchis Palacio, J.R. \& Amigo Pérez, M. (Coords.): El modelo de la Economía del Bien Común. Aplicación en la empresa/organización y casos prácticos, 19-46. Delta Publicaciones, Madrid.

MONTIEL, I. (2008): "Corporate social responsibility and corporate sustainability: Separate pasts, common futures", Organization \& Environment, 21(3), 245-269.

DOI: $10.1177 / 1086026608321329$.

MORA RODRÍGUEZ, A., LÓPEZ MONLLOR, C. \& HILLIARD, I. (2018): “Evaluando los stakeholders de la Economía del Bien Común", CIRIEC-España, Revista de Economía Pública, Social y Cooperativa, 94, 251-283, D0I: 10.7203/CIRIEC-E.94.10458.

MUÑOZ-MARTíN, J. (2013): "Ética empresarial, Responsabilidad Social Corporativa (RSC) y Creación de Valor Compartido (CVC)", Revista de Globalización, Competitividad y Gobernabilidad, 7(3), 76-85. D0I: 10.3232/GCG.2013.V7.N3.05.

NALEBUFF, B.J. \& BRANDENBURGER, A.M. (1996): La co-opétition: une révolution dans la manière de jouer concurrence et coopération, Village Mondial.

OLLÉ-ESPLUGA, S., MUCKENHUBER, J. \& HADLER, M. (2020): “Job Quality in the Economy for the Common Good: conceptualisation and implementation in Austria and Germany", CIRIEC-España, Revista de Economía Pública, Social y Cooperativa, 99, 177-202. D0I: 10.7203/ CIRIEC-E.99.16080.

PFITZER, M.W., BOCKSTETTE, V. \& STAMP, M. (2013): "Innovating for shared value", Harvard Business Review, 91(9), 100-107. https://hbr.org/2013/09/innovating-for-shared-value.

PORTER, M.E. \& KRAMER, M.R. (2006): "The link between competitive advantage and corporate social responsibility", Harvard Business Review, 84(12), 78-92. 
PORTER, M.E. \& KRAMER, M.R. (2011): "The big idea: Creating shared value”, Harvard Business Review, 89(1), 2.

RAWLS, J. (1971): A theory of justice, Harvard University Press, Cambridge.

RIFKIN, J. (2010): La civilización empática: la carrera hacia una conciencia global en un mundo en crisis, Ediciones Paidós Ibérica, Madrid.

RODRÍGUEZ FERNÁNDEZ, C. (2018): “El modelo de la Economía del Bien Común: fundamentos teóricos", En: Sanchis Palacio, J.R \& Amigo Pérez, M. (Coords.), El modelo de la Economía del Bien Común. Aplicación en la empresa/organización y casos prácticos, 3-18. Delta Publicaciones, Madrid.

RODRIK, D. (2011): The globalization paradox: democracy and the future of the world economy, W. W. Norton \& Company, New York.

SALZMANN, O., IONESCU-SOMERS, A. \& STEGER, U. (2005): "The business case for corporate sustainability: literature review and research options", European Management Journal, 23(1), 27-36. DOI: 10.1016/j.emj.2004.12.007.

SANCHIS PALACIO, J.R. \& AMIGO PÉREZ, M. (Coords.) (2018): El modelo de la Economía del Bien Común. Aplicación en la empresa/organización y casos prácticos, Delta Publicaciones, Madrid.

SANCHIS PALACIO, J.R. \& CAMPOS CLIMENT, V. (2018): "Economía del Bien Común y Finanzas Éticas", CIRIEC-España, Revista de Economía Pública, Social y Cooperativa, 93, 241-264. D0I: 10.7203/CIRIEC-E.93.11012.

SANCHIS PALACIO, J.R. \& CAMPOS CLIMENT, V. (2019): "El modelo de la economía del bien común: aproximación desde el enfoque organizativo y el análisis bibliométrico", Estudios Gerenciales, 35(153), 440-450, DOI: 10.18046/j.estger.2019.153.3361.

SEN, A. (1999): On ethics and economics, Oxford University Press, Oxford.

SIEBENHÜNER, B. \& ARNOLD, M. (2007): "Organizational learning to manage sustainable development", Business strategy and the environment, 16(5), 339-353. D0I: 10.1002/bse.579.

STIGLITZ, J.E., SEN, A. \& FITOUSSI, J.P. (2013): Medir nuestras vidas. Las limitaciones del PIB como indicador de progreso, RBA, Barcelona.

VAN MARREWIJK, M. (2003): "Concepts and definitions of CSR and corporate sustainability: Between agency and communion", Journal of Business Ethics, 44(2-3), 95-105. D0I: 10.1023/A\%3A1023331212247

ZAMAGNI, S. (2007): "El bien común en la sociedad posmoderna: propuestas para la acción político-económica", Cultura Económica, 70, 23-43. 